\title{
Dynamical quarks effects on the gluon propagation and chiral symmetry restoration
}

\author{
A. Bashir \\ Universidad Michoacana de San Nicolás de Hidalgo, Edificio C-3, Ciudad Universitaria, \\ Morelia, Michoacán 58040, México. \\ E-mail: adnaneifm.umich.mx
}

\section{A. Raya}

Universidad Michoacana de San Nicolás de Hidalgo, Edificio C-3, Ciudad Universitaria, Morelia, Michoacán 58040, México.

$\&$

Facultad de Física, Pontificial Universidad Católica de Chile, Casilla 306, Santiago 22, Chile

E-mail: raya@ifm.umich.mx

\section{J. Rodríguez-Quintero*}

Departamento de Física Aplicada, Facultad de Ciencias Experimentales, Universidad de Huelva, Huelva 21071, Spain.

$\&$

CAFPE, Universidad de Granada, E-18071, Granda, Spain.

E-mail: jose.rodriguez@dfaie.uhu.es

We exploit the recent lattice results for the infrared gluon propagator with light dynamical quarks and solve the gap equation for the quark propagator. Chiral symmetry breaking and confinement (intimately tied with the analytic properties of QCD Schwinger functions) order parameters are then studied.

QCD-TNT-III-From quarks and gluons to hadronic matter: A bridge too far?,

2-6 September, 2013

European Centre for Theoretical Studies in Nuclear Physics and Related Areas (ECT*), Villazzano, Trento (Italy)

\footnotetext{
* Speaker.
} 


\section{Introduction}

This contribution is mainly devoted to report on the results of Ref. [1], where we dealt with the dynamical quarks effects on the gluon propagation and applied this knowledge to the analysis of the chiral symmetry restoration with increasing number of light fermion flavours. Quantum chromodynamics (QCD) with large number of massless fermion flavors has seen a resurgence of interest due to its connection with technicolor models, originally proposed by Weinberg and Susskind [2], which fall into the category of Beyond the Standard Model Theories. They possess intrinsically attractive features. They do not resort to fundamental scalars to reconcile local gauge symmetry with massive mediators of interaction and have close resemblance with well-studied fundamental strong interactions, i.e., QCD. However, their simple versions do not live up to the experimental electroweak precision constraints, in particular the ones related to flavor changing neutral currents. Walking models containing a conformal window and an infrared fixed point can possibly cure this defect and become phenomenologically viable [3]. This scenario motivates the investigation of QCD for similar characteristics. One looks for such behavior of QCD for large number of light flavors albeit less than the critical value where asymptotic freedom sets in, i.e., $N_{f}^{c_{1}}=16.5$, a Nobel prize winning result known since the advent of QCD, [4]. Just as $N_{f}$ dictates the peculiar behavior of QCD in the ultraviolet, we expect it to determine the onslaught of its emerging phenomena in the infrared, i.e., chiral symmetry breaking and confinement.

Whereas the self interaction of gluons provides anti-screening, the production of virtual quarkantiquark pairs screens and debilitates the strength of this interaction of non abelian origin. For real QCD, light flavors are small in number and hence yield to the gluonic influence which triggers confinement and chiral symmetry breaking. One needs to establish if there is another critical value $N_{f}^{c_{2}}<N_{f}^{c_{1}}$ which can sufficiently dilute the gluon-gluon interactions to restore chiral symmetry and deconfine color degrees of freedom. Such a phase transition lies at the non perturbative boundary of the interactions under scrutiny and hence we cannot expect to extract sufficiently reliable information from multiloop calculations of the QCD $\beta$-function. Purely non perturbative techniques are required to tackle the problem. Lattice studies in the infrared indicate that just below $N_{f}^{c_{1}}$, chiral symmetry remains unbroken and color degrees of freedom are unconfined [5]. Below this conformal window, for an $8<N_{f}^{c_{2}}<12$, the evolution of the beta function in the infrared is such that QCD enters the phase of dynamical mass generation as well as confinement.

Modern lattice analyses for this matter appear to strongly argue in favour of a restoration for the chiral symmetric phase taking place somewhere between $N_{f} \sim 8$ and $N_{f} \sim 10$ [6,7]. In particular, the authors of ref. [7], with their study of the meson spectrum in lattice QCD with eight light flavours using the Highly Improved Staggered Quark action, gathered some striking evidences that $N_{f}=8$ QCD still lies in the broken chiral symmetry phase but, at the sime time, suffering the effects from a remnant of the infrared conformality (a large anomalous dimension for the quark mass renormalization constant) indicating that the unbroken phase is recovered near above $N_{f} \sim 8$. In the present work, we intend to combine the Schwinger-Dyson machinery, well adjusted to account for QCD phenomenology in the pion sector, with the very last lattice data including twisted-mass dynamical light flavours in order to provide with a model for the chiral restoration mechanism, in quantitative agreement with the above mentioned lattice studies. 


\section{Chiral phase transition picture from Schwinger-Dyson and Lattice gluon propagators}

In continuum, Schwinger-Dyson equations (SDEs) of QCD provide an ideal framework to study its infrared properties, [8]. These are the fundamental equations of any quantum field theory, linking all its defining Green functions to each other through intricately coupled nonlinear integral equations. As their formal derivation through variational principle makes no appeal to the weakness of the interaction strength, they naturally connect the perturbative ultraviolet physics with its emerging non perturbative properties in the infrared sector within the same framework. The simplest two-point quark propagator is a basic object to analyze dynamical chiral symmetry breaking and confinement. Within the formalism of the SDEs, the inverse quark propagator can be expressed as

$$
S^{-1}(p)=\mathscr{Z}_{2}(i \gamma \cdot p+m)+\Sigma(p)
$$

where $\Sigma(p)$ is the quark self energy:

$$
\Sigma(p)=\mathscr{Z}_{1} \int \frac{d^{4} q}{(2 \pi)^{4}} g^{2} \Delta_{\mu v}(p-q) \frac{\lambda^{a}}{2} \gamma_{\mu} S(q) \Gamma_{v}^{a}(q, p),
$$

where $\mathscr{Z}_{1}=\mathscr{Z}_{1}\left(\mu^{2}, \Lambda^{2}\right)$ and $\mathscr{Z}_{2}=\mathscr{Z}_{2}\left(\mu^{2}, \Lambda^{2}\right)$ are the renormalization constants associated respectively with the quark-gluon vertex and the quark propagator. $\Lambda$ is the ultraviolet regulator and $\mu$ is the renormalization point. The solution to this equation is

$$
S^{-1}(p)=\frac{i \gamma \cdot p+M\left(p^{2}\right)}{Z\left(p^{2}, \mu^{2}\right)}
$$

where $Z\left(p^{2}, \mu^{2}\right)$ is the quark wavefunction renormalization and the quark mass function $M\left(p^{2}\right)$ is renormalization group invariant. This equation involves the quark-gluon vertex $\Gamma_{v}^{a}(q, p)$ and the gluon propagator $\Delta_{\mu v}(p-q)$. Very much attention will be paid in the next subsection to the twopoint function as a crucial input to study the quark propagator. Here, in the following, the only other ingredient, the three-point quark-gluon vertex $\Gamma_{v}^{a}(q, p)$, will be briefly discussed.

Significant advances have been made in pinning it down through its key attributes in the ultraviolet and infrared domains [9]. More recently, the seeds of the most general ansatz for the fermion-boson vertex appeared in [10] and its full blown extension was presented in [11]. Furthermore, given the general nature of constraints and the simplicity of the construction, a straightforward extension of this approach is expected to yield an ansatz adequate to the task of representing the dressed-quark-gluon vertex. Before this is achieved, we restrict ourselves to an effective though efficacious approach. Following the lead of Maris et. al. [12], we employ the following suitable ansatz which has sufficient integrated strength in the infrared to achieve dynamical mass generation:

$$
\mathscr{Z}_{1} g^{2} \Delta_{\mu v}(p-q) \Gamma_{v}(p, q) \rightarrow g_{\text {eff }}^{2}\left(q^{2}\right) \Delta_{\mu v}^{N}\left(p-q, N_{f}\right) \frac{\lambda^{a}}{2} \gamma_{v}
$$

where

$$
\Delta_{\mu v, N_{f}}^{N}(q)=\frac{D\left(q^{2}, N_{f}\right)}{q^{2}}\left[\delta_{\mu v}-\frac{q_{\mu} q_{v}}{q^{2}}\right]
$$


In the next subsection, the nonperturbative gluon dressing function, $D\left(q^{2}, N_{f}\right)$, will appear modelled and accounting thus for the effects from the dynamical light flavours on the gluon propagation. Concerning the effective coupling, $g_{\text {eff }}$, it has been chosen to be

$$
g_{\mathrm{eff}}^{2}\left(q^{2}\right) D\left(q^{2}, N_{f}\right)=\frac{D\left(q^{2}, N_{f}\right)}{D\left(q^{2}, 2\right)} g_{\mathrm{MT}}^{2}\left(q^{2}\right)
$$

where $g_{M T}^{2}$ stands for the effective coupling ansatz proposed by Maris et. al. in ref. [12] and built there to incorporate also the nonperturbative information from the gluon propagator (as it is assumed to multiply the tree-level one) . Thus, Eq. (2.6) for two degenerate light fermion flavours allows to reproduce correctly the static as well as dynamic properties of mesons below $1 \mathrm{GeV}$ (see for example review [13] and references therein) while, by fixing the right number of flavours in the perturbative tail of $g_{M T}^{2}$, it also reproduces the appropriate ultraviolet behaviour, for any flavour number.

\subsection{Modelling the flavour behaviour for the gluon propagator}

The second ingredient needed to solve Eq. (2.1) with $(2.2,2.3)$ is the two-point gluon Green function, $\Delta_{\mu v}$, that has been the object of a patient effort, spanning several decades, addressed to unravel its infrared behaviour. Lattice as well as SDE studies have finally converged on its massive or so called decoupling solution (see for example [14]). After the gluon propagator solution in the quenched approximation has been chiselled, we now have the first quantitatively reliable glimpses of its quark flavor dependence by incorporating $N_{f}=0,2$ light dynamical quark flavors ${ }^{1}$ and 2+1+1 (2 light degenerate quarks, with masses ranging from 20 to $50[\mathrm{MeV}]$, and two non degenerate flavors for the strange and the charm quarks, with their respective masses set to 95 $[\mathrm{MeV}]$ and $1.51[\mathrm{GeV}])$ [21]. As we demonstrate shortly, in this last $2+1+1$ case, the number of light quarks corresponds effectively to 3 . This is exactly the result derived from the recently developed "partially unquenched" approach to incorporate flavor effects in the gluon SDE, [22]. Their work is in agreement with one of [21] when the charm flavor is assumed to decouple from gluons. It should not be worthless to remark that the same $N_{f}=2+1+1$ gauge fields have been also used to compute the running strong coupling and estimate accurately the value of $\Lambda_{\overline{\mathrm{MS}}}$, in very good agreement with experiments [23].

Moreover, our modern understanding of the flavor dependence of the gluon propagator provides us with the solid basis to use the following non perturbative model [24]:

$$
D\left(q^{2}\right)=\frac{z\left(\mu^{2}\right) q^{2}\left(q^{2}+M^{2}\right)}{q^{4}+q^{2}\left(M^{2}-13 g^{2}\left\langle A^{2}\right\rangle / 24\right)+M^{2} m_{0}^{2}}
$$

to describe the gluon dressing renormalized in MOM scheme at $q^{2}=\mu^{2}$. This model is based on the tree-level gluon propagator obtained with the Renormalized Gribov-Zwanziger (RGZ) action [25] which have been shown to describe properly the lattice data in the infrared sector (see refs. [26, 24]). The overall factor $z\left(\mu^{2}\right)$ is introduced to guarantee the multiplicative MOM renormalization prescription, namely, $D\left(\mu^{2}\right)=1$, and implies no physical consequence as the effective coupling,

\footnotetext{
${ }^{1}$ The dynamical flavors have been generated, within the framework of ETM collaboration [15, 16, 17, 18], with the mass-twisted lattice action [19], while $N_{f}=0$ data have been borrowed from [20].
} 

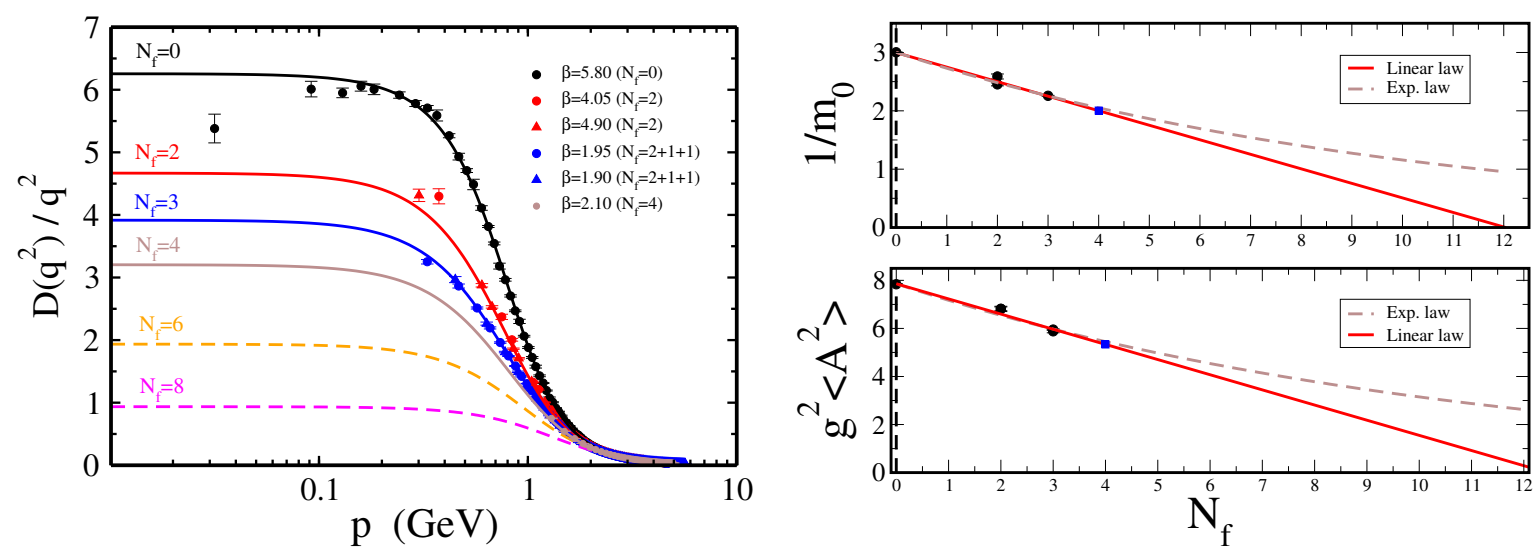

Figure 1: (Left) Lattice gluon propagator data in terms of momenta for different number of fermion flavors and fits with Eq. (2.7) and the parameters of Eqs. (2.9,2.10). (Right) Parameters $g^{2}<A^{2}>$ and $1 / m_{0}^{2}$ in terms of the numbers of flavors and the fits with Eqs. $(2.9,2.10)$. The blue squares stand for the extrapolated results at $N_{f}=4$ we used for Fig. 2 .

$g_{\text {eff }}$, is further adjusted to reproduce properly the meson phenomenology. Then, we apply Eq. (2.7) to reproduce the gluon propagator lattice data analyzed in Ref. [21] and thus fit its mass parameters. $M^{2}$ is related to the condensate of auxiliary fields, emerging merely to preserve locality for the RGZ action. A free fit of the lattice data suggests that it does not depend on the number of fermion flavors (we find $M^{2}=4.85\left[\mathrm{GeV}^{2}\right]$ ). Dimension two gluon condensate $\left\langle A^{2}\right\rangle$, [27], and

$$
m_{0}^{2}=z\left(\mu^{2}\right) \lim _{q^{2} \rightarrow 0} \frac{q^{2}}{D\left(q^{2}\right)}
$$

are flavor dependent and we look for their best fits. In order to cover a wide range of possibilities within reason, we assume their evolution with the flavor number to be driven either by a simple linear scaling law

$$
\begin{aligned}
m_{0}^{-1}\left(N_{f}\right) & =m_{0}^{-1}(0)\left(1-A N_{f}\right) \\
g^{2}\left\langle A^{2}\right\rangle\left(N_{f}\right) & =g^{2}\left\langle A^{2}\right\rangle(0)\left(1-B N_{f}\right),
\end{aligned}
$$

as data appear to suggest, or by an exponential law

$$
\begin{aligned}
m_{0}^{-1}\left(N_{f}\right) & =m_{0}^{-1}(0) e^{-A N_{f}} \\
g^{2}\left\langle A^{2}\right\rangle\left(N_{f}\right) & =g^{2}\left\langle A^{2}\right\rangle(0) e^{-B N_{f}},
\end{aligned}
$$

which allows for the possibility that the gluon propagator becomes infinitely massive only when the number of light quark flavors tends to infinity. The best-fit of the $m_{0}$ and $g^{2}\left\langle A^{2}\right\rangle$ from lattice data will require $m_{0}(0)=0.333 \mathrm{GeV}$ and $g^{2}\left\langle A^{2}\right\rangle(0)=7.856$, in both cases, $A=0.083$ and $B=$ 0.080, for the linear case, and $A=0.095$ and $B=0.091$, for the exponential one. Eq. (2.7) now provides prediction for the gluon propagator for arbitrarily large $N_{f}$, as can be seen in the right plot Fig. 1, while the right one shows the corresponding gluon propagator along with the lattice data superimposed [21]. We also include some very recent gluon propagator data obtained from 
lattice simulations with four degenerate light twisted-mass flavors ${ }^{2}$. These new data are rather well described by Eq. (2.7) evaluated for the mass parameters extrapolated to $N_{f}=4$ with Eq. (2.9) (see the zoomed plot in Fig. 2). This observation strongly supports that $N_{f}=2+1+1$ gluon data indeed correspond to three light flavors.

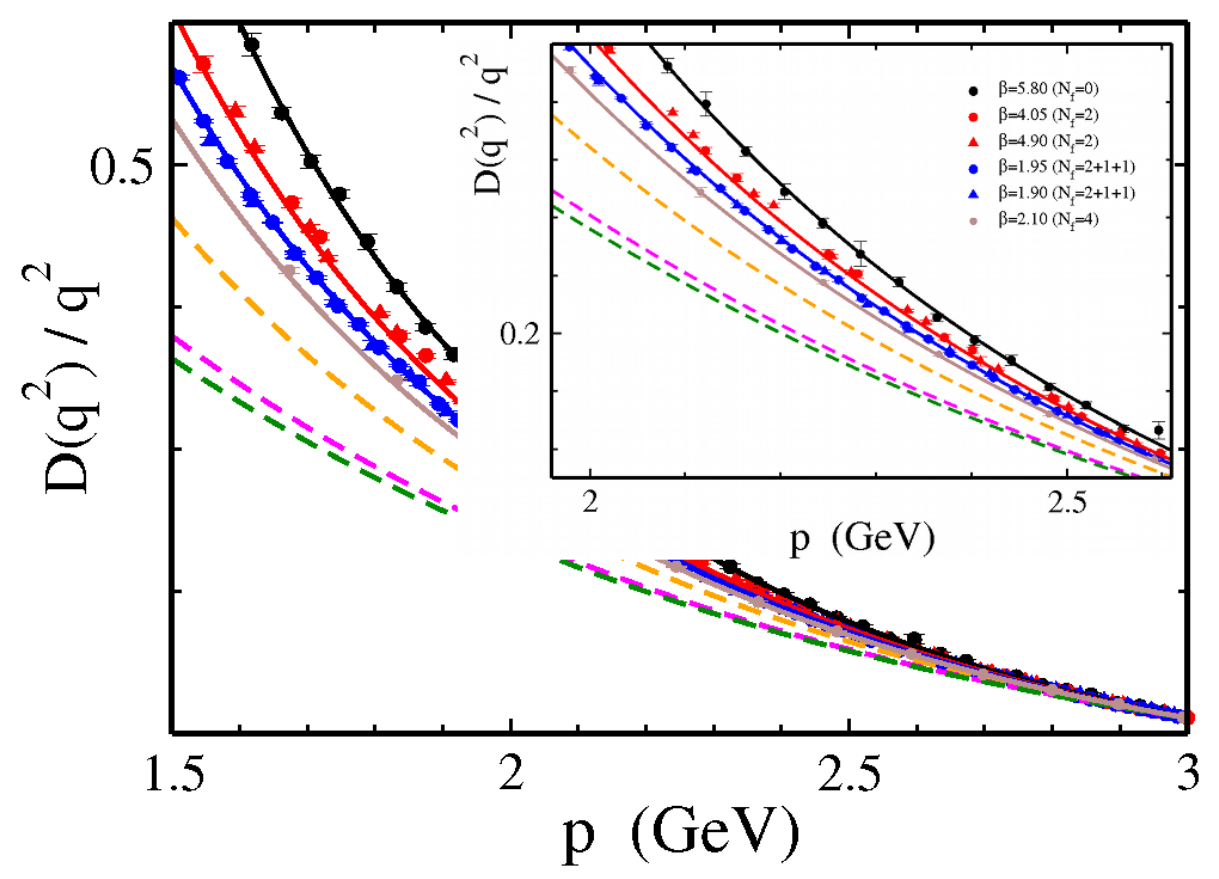

Figure 2: The same of the left plot of Fig. 1 but only with the parameters for the linear case and incorporating new small-volume lattice data for 4 degenerate fermion flavors.

Thus, we can efficaciously model the dilution of the gluon-gluon interactions with increasing flavor number in order to study the chiral restoration mechanism. We can now employ the gap equation to provide quantitative details of chiral symmetry breaking in terms of the quark mass function for an increasing number of light quarks.

\subsection{Results}

In the following, we mostly discuss the results obtained by employing the linear law and state the effect of exponential extrapolation afterwards. Note that we have not considered the flavor dependence which would arise from the quark-gluon vertex (no explicit handle on this dependence is available at the moment). Otherwise said, as can be seen from Eq. (2.6), we take the effective coupling $g_{\text {eff }}$, in the IR, to depend on $N_{f}$ only through the gluon dressing function. The latter can be fairly justified by the results of [21] (see Eq.(5.2)) which suggest that an effective coupling can be constructed such that there is an absence of any flavor dependence in the infrared region, more precisely starting from $q^{2} \lesssim 1 \mathrm{GeV}^{2}$.

Once the quark mass function is available for varying light quark flavors (see Fig. 3 for the linear case), one can investigate any of the interrelated order parameters, namely, the Euclidean

\footnotetext{
${ }^{2}$ The gluon propagator lattice data for 4 light flavors have been borrowed from ETMC [28]. Simulated at small volumes, they are only available for momenta above $1.25 \mathrm{GeV}$ and hardly allow for a fit with Eq. (2.7). Nevertheless, they can be used to check our modelling of the flavor evolution.
} 


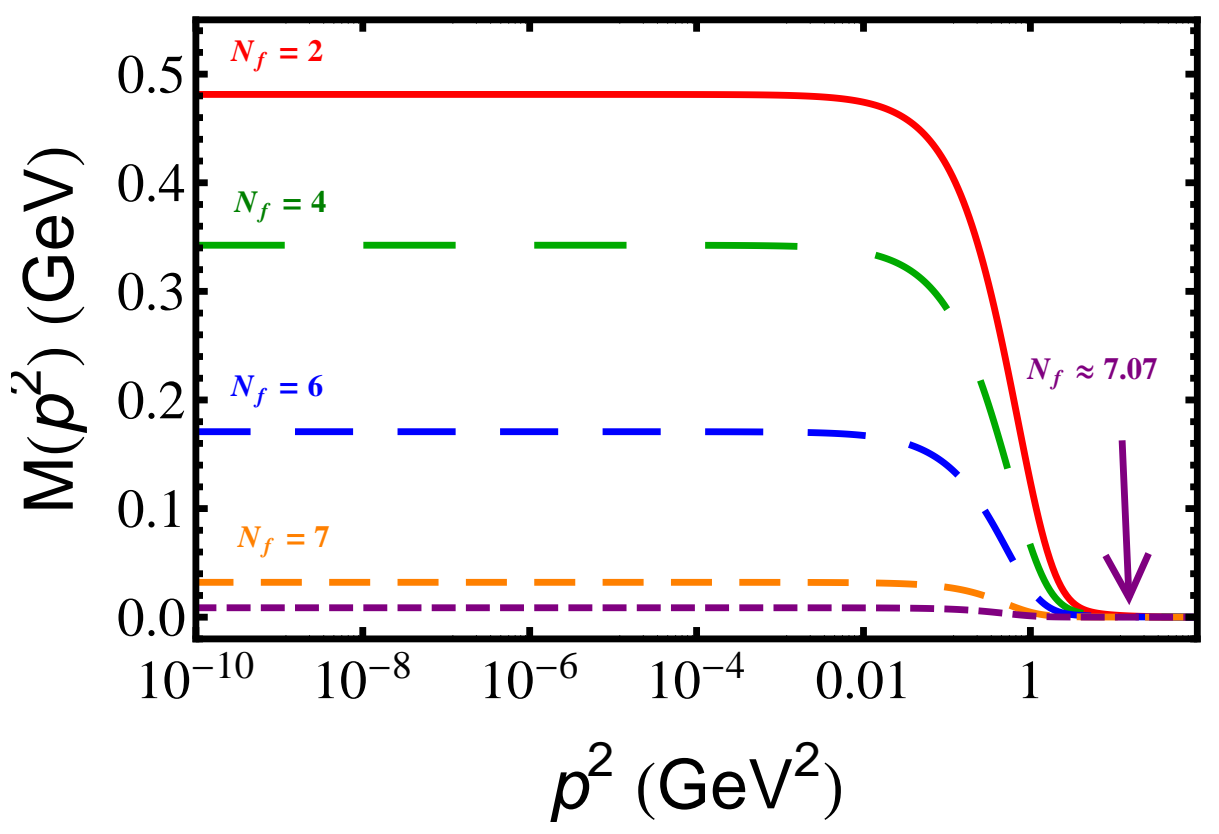

Figure 3: The quark mass function diminishes height for increasing light quark flavors (here with Eq. (2.9) Above $N_{f} \approx 7.07$, only the chirally symmetric solution exists.

pole mass defined as $m_{\text {dyn }}^{2}+M^{2}\left(p^{2}=m_{\text {dyn }}^{2}\right)=0$, the quark-antiquark condensate which is obtained from the trace of the quark propagator or the pion leptonic decay constant $f_{\pi}$ defined through the Pagel-Stokar equation [29], or through considering the residue at the pion pole of the meson propagator. Each of these quantities involves the quark wave-function renormalization, the mass function and/or its derivatives and is hence calculable from the solution for the full quark propagator. Moreover, these order parameters can help to locate the critical number of flavors above which chiral symmetry is restored.

We investigate these three order parameters and choose to present here the Euclidean pole mass of the quark in Fig. 4 for the linear (exponential) case and show that, at a critical value of about $N_{f}^{c} \approx 7.1\left(N_{f}^{c} \approx 9.4\right)$, chiral symmetry appears restored. The phase transition appears second order, described by the following mean field behavior (solid lines in Fig. 4) :

$$
m_{\mathrm{dyn}} \sim \sqrt{N_{f}^{c_{2}}-N_{f}}
$$

It has been established that confinement is related to the analytic properties of QCD Schwinger functions which are the Euclidean space Green functions, namely, propagators and vertices. One deduces from the reconstruction theorem [30] that the only Schwinger functions which can be associated with expectation values in the Hilbert space of observables; namely, the set of measurable expectation values, are those that satisfy the axiom of reflection positivity. When that happens, the real-axis mass-pole splits, moving into pairs of complex conjugate singularities. No mass-shell can be associated with a particle whose propagator exhibits such singularity structure. We define the 


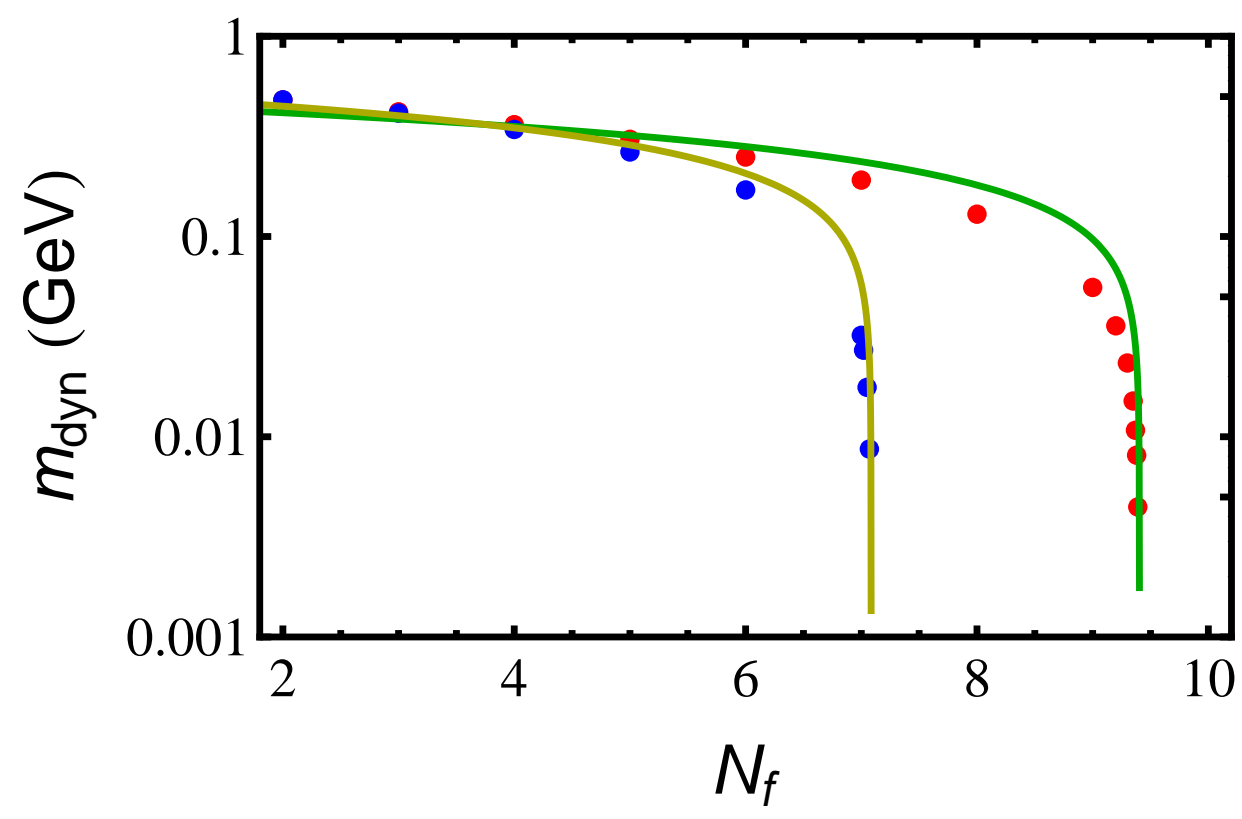

Figure 4: Quark pole mass in the Euclidean space clearly demonstrates that chiral symmetry is restored above a critical number of quark flavors. Blue (red) points correspond to linear (exponential) case. The solid line is the mean-field scaling, Eq.(2.11)

following Schwinger function:

$$
\Delta(t)=\int d^{3} x \int \frac{d^{4} p}{(2 \pi)^{4}} \mathrm{e}^{i\left(p_{4} t+\mathbf{p} \cdot \mathbf{x}\right)} \sigma_{s}\left(p^{2}\right)
$$

to study the analytic properties of the quark propagator; where $\sigma_{s}\left(p^{2}\right)$ is the scalar term for the quark propagator in Eq. (2.3), that can be written in terms of the quark wavefunction renormalization and mass function as $Z\left(p^{2}, \mu^{2}\right) M\left(p^{2}\right) /\left(p^{2}+M\left(p^{2}\right)\right)$. One can show that if there is a stable asymptotic state associated with this propagator, with a mass $m$, then

$$
\Delta(t) \sim \mathrm{e}^{-m t},
$$

whereas two complex conjugate mass-like singularities, with complex masses $\mu=a \pm i b$ lead to an oscillating behavior of the sort

$$
\Delta(t) \sim \mathrm{e}^{-a t} \cos (b t+\delta)
$$

for large $t$, [31]. Fig. 5 analyzes this function for varying $N_{f}$, in the linear extrapolation case. The existence of oscillations clearly demonstrates that the quarks correspond to a confined excitation for small $N_{f}$. With increasing $N_{f}$, the onslaught of oscillations moves towards higher values of $t$ and eventually never takes place above a critical $N_{f}$ when quarks deconfine and correspond to a stable asymptotic state. As an order parameter of confinement, we therefore employ $v\left(N_{f}\right)=1 / \tau_{1}\left(N_{f}\right)$, where $\tau_{1}\left(N_{f}\right)$ is the location of the first singularity, [32]. The first oscillation is pushed to infinity when confinement is lost. It is notable that when the dynamically generated mass approaches zero, $v\left(N_{f}\right)$ diminishes rapidly (see right plot of Fig. 5). This highlights the intimate connection between 

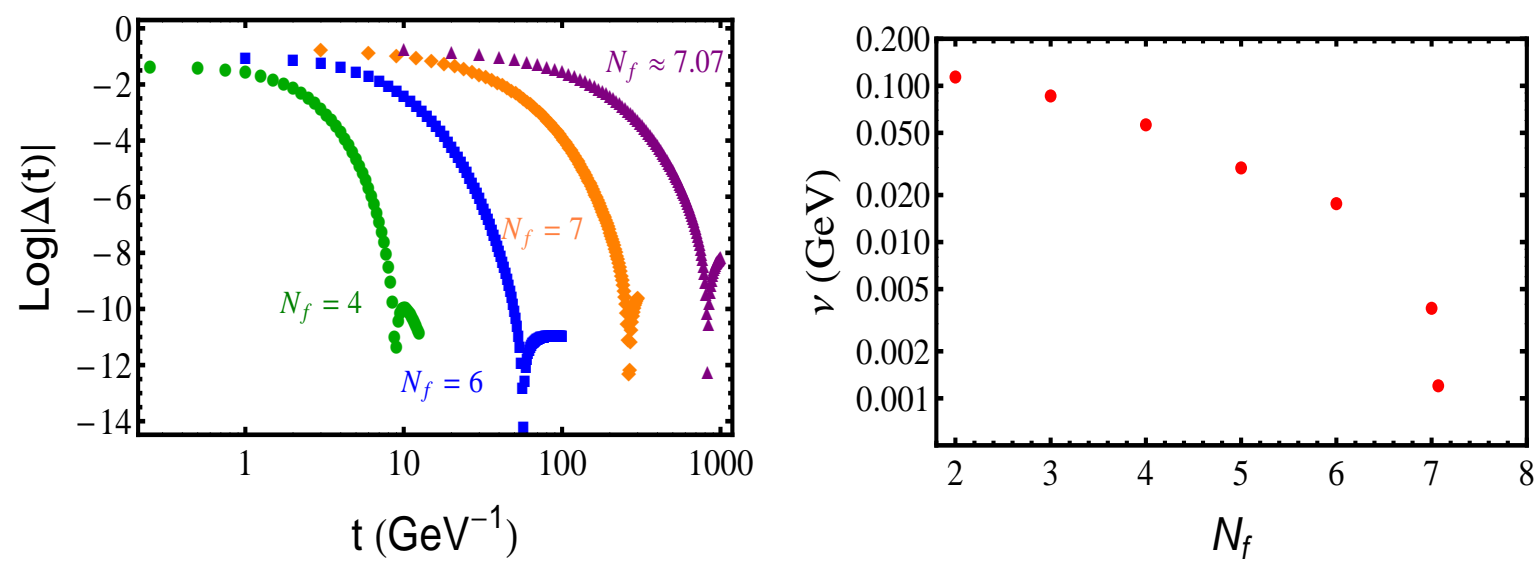

Figure 5: (Left) Spatially averaged Euclidean space 2-point Schwinger function $\Delta(t)$ develops oscillations for large times which corresponds to the non-existence of asymptotically stable free quark states. For sufficiently large values of $N_{f}$, the first minimum of these oscillations is pushed all the way to infinity, thus ensuring the existence of a pole on the time-like axis, a property of free particle propagators. (Right) The order parameter for confinement $v\left(N_{f}\right)=1 / \tau_{1}\left(N_{f}\right)$, where $\tau_{1}\left(N_{f}\right)$ is the location of the first zero of Eq. (2.12) Comparison with Fig. 4 suggests that quarks get deconfined when chiral symmetry is restored.

chiral symmetry restoration and deconfinement. In fact, within our numerical accuracy, $N_{f}^{c}$ is found to be the same for both the transitions.

The results with the exponential and linear flavor extrapolations are qualitatively the same, leading to identical conclusions. They only quantitatively differ by the critical flavor numbers, although both are pretty much in the same ballpark: $N_{f}^{c} \simeq 7.1$ and $N_{f}^{c} \simeq 9.4$. Note that both the parameterizations, so far apart as to have an infinitely massive gluon at $N_{f} \approx 12$ or $N_{f} \Rightarrow \infty$, restore chiral symmetry and trigger deconfinement at so similar value of light quark flavors.

\section{Conclusions}

We have benefited from the latest lattice result for the quark flavor dependence of the gluon propagator in the infrared, as well as from a RGE-grounded nonperturbative model for this IR gluon propagator, in order to perform a Poincare-covariant SDE analysis for the quark propagator. This provided with an efficacious model for dilution of the gluon-gluon interaction with increasing number of light quarks and we applied it to study the evolution with the light-flavors number of the quark chiral behaviour. A nonperturbative picture for the chiral symmetry restoration mechanism emerges thus from this analysis. The quantitative analysis, following this approach, hints towards chiral symmetry restoration in QCD when the number of light quark flavors exceeds a critical value of $N_{f}^{c_{2}} \approx 8.2 \pm 1.2$. This is in perfect agreement with the state-of-the-art for the direct lattice investigations on the chiral symmetry restoration in QCD $[6,7]$ and supports that the model presented here for the chiral restoration mechanism is properly capturing most of the relevant physics for the problem we deal with. 
Furthermore, we have also studied an order parameter for the QCD confinement-deconfinement transition, which is based on the analytic properties of the quark two-point Schwinger function, and located numerically the critical point at the same number of flavors as chiral symmetry restoration took place. That chiral symmetry appears restored when quarks get deconfined, within the approach we followed, highlights the intimate connection betweem both fundamental phenomena of QCD. This is a main result of this work.

Acknowledgments We acknowledge D. Schaich for a fruitful communication. This work was supported by the grants: CIC, UMICH, Mexico, 4.10 and 4.22, CONACyT (Mexico) 82230 and 128534, and MINECO (Spain) research project FPA2011-23781.

\section{References}

[1] A. Bashir, A. Raya and J. Rodriguez-Quintero, Phys. Rev. D 88, 054003 (2013); arXiv:1302.5829 [hep-ph].

[2] S. Weinberg, Phys. Rev. D 13, 974 (1976); S. Weinberg, Phys. Rev. D 19, 1277 (1979); L. Susskind, Phys. Rev. D 20, 2619 (1979).

[3] B. Holdom, Phys. Lett. B 150, 301 (1985); K. Yamawaki, M. Bando and K. Matumoto, Phys. Rev. Lett. 56, 1335 (1986); T. W. Appelquist, D. Karabali and L. C. R. Wijewardhana, Phys. Rev. Lett. 57, 957 (1986).

[4] D. J. Gross and F. Wilczek, Phy. Rev. Lett. 30, 1343 (1973); H. D. Politzer, Phy. Rev. Lett. 30, 1346 (1973).

[5] T. Appelquist et. al., Phys. Rev. Lett. 104, 071601 (2010); T. Appelquist, G. T. Fleming and E. T. Neil, Phys. Rev. D 79, 076010 (2009); Z. Fodor et. al., Phys. Lett. B 681, 353 (2009); K.-I. Nagai et. al., Phys. Rev. D 80, 074508 (2009); L. Del Debbio et. al., Phys. Rev. D 82, 014510 (2010); A. Hasenfratz, Phys. Rev. D 82, 014506 (2010).

[6] M. Hayakawa, K.-I. Ishikawa, Y. Osaki, S. Takeda, S. Uno, N. Yamada, Phys. Rev. D 83074509 (2011); "Approaching Conformality with Ten Flavors", T. Appelquist, R. C. Brower, M. I. Buchoff, M. Cheng, S. D. Cohen, G. T. Fleming, J. Kiskis, M. Lin, H. Na, E. T. Neil, J. C. Osborn, C. Rebbi, D. Schaich, C. Schroeder, G. Voronov, P. Vranas, arXiv:1204.6000 [hep-ph] (2012) ; Y. Iwasaki, "Conformal Window and Correlation Functions in Lattice Conformal QCD", e-Print: arXiv:1212.4343 [hep-lat] (2012); "Scale-dependent Mass Anomalous Dimension from Dirac Eigenmodes", A. Cheng, A. Hasenfratz, G. Petropoulos, D. Schaich, arXiv:1301.1355 [hep-lat] (2013).

[7] Y. Aoki, T. Aoyama, M. Kurachi, T. Maskawa, K-I Nagai, H. Ohki, A. Shibata, K. Yamawaki, T. Yamazaki; "Walking Signals in Nf=8 QCD on the Lattice", arXiv:1302.6859 [hep-lat] (2013).

[8] F. Dyson, Phys. Rev. 75, 1736 (1949); J. S. Schwinger, Proc. Nat. Acad. Sci. 37, 452 (1951); J. S. Schwinger, Proc. Nat. Acad. Sci. 37, 455 (1951).

[9] J. S. Ball and T-W. Chiu, Phys. Rev. D 22, 2542 (1980); D. C. Curtis and M. R. Pennington, Phys. Rev. D 42, 4165 (1990); Z. Dong, H. J. Munczek and C. D. Roberts, Phys. Lett. B 33, 536 (1994); A. Bashir and M. R. Pennington, Phys. Rev. D 50, 7679 (1994); A. Kizilersu and M. R. Pennington, Phys. Rev. D 79125020 (2009).

[10] A. Bashir, A. Raya, S. Sánchez-Madrigal, Phys. Rev. D 84, 036013 (2011). 
[11] A. Bashir, R. Bermudez, L. Chang, C. D. Roberts, Phys. Rev. C 85, 045205 (2012).

[12] P. Maris, C. D. Roberts and P. C. Tandy, Phys. Lett. B 420, 267 (1998); P. Maris and C. D. Roberts, Phys. Rev. C 583659 (1998).

[13] A. Bashir, L. Chang, I. C. Clot, B. El-Bennich, Y-X. Liu, C. D. Roberts, P. C. Tandy, Commun. Theor. Phys. 58, 79, (2012).

[14] I. L. Bogolubsky, E. M. Ilgenfritz, M. Muller-Preussker and A. Sternbeck, Phys. Lett. B 676, 69 (2009); A. C. Aguilar, D. Binosi, and J. Papavassiliou, Phys. Rev. D 78, 025010 (2008); P. Boucaud, J. Leroy, A. L. Yaouanc, J. Micheli, O. Péne, and J. Rodríguez-Quintero, J. High Energy Phys. 06, 099 (2008); D. Dudal, J. A. Gracey, S. P. Sorella, N. Vandersickel, and H. Verschelde, Phys. Rev. D 78, 065047 (2008).

[15] R. Baron, P. Boucaud, J. Carbonell, A. Deuzeman, V. Drach, F. Farchioni, V. Gimenez and

G. Herdoiza et al., JHEP 1006, 111 (2010).

[16] R. Baron et al. [ETM Collaboration], PoS LATTICE 2010, 123 (2010).

[17] B. Blossier et al. [ETM Collaboration], Phys. Rev. D 82, 034510 (2010).

[18] B. Blossier, P. .Boucaud, M. Brinet, F. De Soto, X. Du, M. Gravina, V. Morenas and O. Pene et al., Phys. Rev. D 85, 034503 (2012),

[19] R. Frezzotti et al. [Alpha Collaboration], JHEP 0108, 058 (2001).

[20] I. L. Bogolubsky, E. M. Ilgenfritz, M. Muller-Preussker and A. Sternbeck, PoS LAT 2007, 290 (2007).

[21] A. Ayala, A. Bashir, D. Binosi, M. Cristoforetti and J. Rodriguez-Quintero, Phys. Rev. D 86, 074512(2012).

[22] A. C. Aguilar, D. Binosi and J. Papavassiliou, Phys. Rev. D 86 (2012) 014032 [arXiv:1204.3868 [hep-ph]].

[23] B. Blossier, P. .Boucaud, M. Brinet, F. De Soto, X. Du, V. Morenas, O. Pene and K. Petrov et al., Phys. Rev. Lett. 108 (2012) 262002 [arXiv:1201.5770 [hep-ph]]; B. Blossier et al. [ETM Collaboration], arXiv:1310.3763 [hep-ph].

[24] D. Dudal, O. Oliveira and J. Rodriguez-Quintero, Phys. Rev. D 86, 105005 (2012).

[25] D. Dudal, J. A. Gracey, S. P. Sorella, N. Vandersickel and H. Verschelde, Phys. Rev. D 78 (2008) 065047 [arXiv:0806.4348 [hep-th]].

[26] D. Dudal, O. Oliveira and N. Vandersickel, Phys. Rev. D 81 (2010) 074505 [arXiv:1002.2374 [hep-lat]].

[27] P. Boucaud, A. Le Yaouanc, J. P. Leroy, J. Micheli, O. Pene and J. Rodriguez-Quintero, Phys. Lett. B 493 (2000) 315 [hep-ph/0008043]; F. V. Gubarev and V. I. Zakharov, Phys. Lett. B 501 (2001) 28 [hep-ph/0010096]; K. -I. Kondo, Phys. Lett. B 514 (2001) 335 [hep-th/0105299]; H. Verschelde, K. Knecht, K. Van Acoleyen and M. Vanderkelen, Phys. Lett. B 516 (2001) 307 [hep-th/0105018]; D. Dudal, H. Verschelde and S. P. Sorella, Phys. Lett. B 555 (2003) 126 [hep-th/0212182]; E. Ruiz Arriola, P. O. Bowman and W. Broniowski, Phys. Rev. D 70 (2004) 097505 [hep-ph/0408309].

[28] P. .Boucaud, M. Brinet, F. De Soto, V. Morènas, O. Pène, K. Petrov and J. Rodríguez-Quintero, arXiv:1312.1514 [hep-lat].

[29] H. Pagels and S. Stokar, Phys. Rev. D 202947 (1979). 
[30] R. F. Streater and A. S. Wightman, "PCT, spin and statistics, and all that," (1989); J. Glimm and A. Jaffee, "Quantum Physics. A Functional Point of View," Springer-Verlag, New York(1981).

[31] P. Maris, Phys. Rev. D 526087 (1995).

[32] A. Bashir, A. Raya, S. Sánchez-Madrigal, C.D. Roberts, Few Body Sys. 46, 229 (2009). 